\title{
Gamma Radiation Induced Modification on Optical Gap of Polycarbonate Detectors
}

\author{
Yasser Saad Rammah \\ Department of Physics, Faculty of Science, Menoufia University, Shebin El-Koom, Egypt
}

Email address:

dr_yasser1974@yahoo.com, dryasser1974@gmail.com

To cite this article:

Yasser Saad Rammah. Gamma Radiation Induced Modification on Optical Gap of Polycarbonate Detectors. American Journal of Optics and Photonics. Vol. 5, No. 3, 2017, pp. 24-29. doi: 10.11648/j.ajop.20170503.11

Received: September 3, 2017; Accepted: September 27, 2017; Published: September 30, 2017

\begin{abstract}
Exposure of polycarbonate detectors to high $\gamma$ - ray doses causes a great effect on their structural. Samples of Makrofol DE 1-1 nuclear track detectors (NTDs) were irradiated with gamma doses in the range of 1-100 kGy using 1.25 MeV ${ }^{60} \mathrm{Co}$ source of dose rate $4 \mathrm{kGy} / \mathrm{h}$. The optical characterization of these detectors have been studied through the measurements of UV-visible absorption spectra of blank and $\gamma$-irradiated samples. The UV-visible spectra of $\gamma$ - irradiated samples revealed color centers in the range (310-320 nm). Direct and indirect allowed optical band gap energies, refractive index (n), the number of carbon atoms per conjugated length $(\mathrm{N})$, and the number of carbon atoms per cluster $(\mathrm{M})$ for pristine and $\gamma$-irradiated samples were determined. Results revealed that optical band gaps decrease, while $\mathrm{n}, \mathrm{N}$, and M increase with increasing of $\gamma$ irradiated doses. This study sheds light on the basis of $\gamma$-interaction mechanisms with the polymeric detectors and use of Makrofol DE 1-1 in gamma dose determination.
\end{abstract}

Keywords: Makrofol DE 1-1 SSNTDs, $\gamma$-irradiation, UV-visible Spectra, Optical Band Gap Energy

\section{Introduction}

In the last few decades the solid-state nuclear track detectors (SSNTDs) have proven to be one of the most efficient, useable and recommended group of detectors available in marketing [1-8]. They acquire many advantages over the others, such as easily processional, low cost in manufcturing, excellent surface transeparency, high effeciency in ion-regestiration, etc. Exposure of SSNTDs to low LET radiations (X-rays, electrons and gamma rays) influences the chemicals and structural properties of these polymers. Interactions of LLET with such detectors produce changes in the bulk material leading to bond breaking, chain scission, ionizing and exciting species, free radicals, etc. Several authors have studied the effect of interaction of different kinds of ions, such as neutrons and electrons on plastic detectors $[9,10]$.

In this study, the optical characterization and the carbonaceous clusters of $\gamma$-irradiated Makrofol DE 1-1 detectors have been carried out through the measurements of the optical gap energies, refractive index (n), the number of carbon atoms per conjugated length $(\mathrm{N})$, and the number of carbon atoms per cluster (M).

\section{Materials and Methods}

Makrofol DE 1-1 (Bisphenol-A polycarbonate) (NTDs) of chemical composition $\left[\mathrm{C}_{16} \mathrm{H}_{14} \mathrm{O}_{3}\right]$ with thickness of $175 \mu \mathrm{m}$ and density $1.2 \mathrm{~g} / \mathrm{m}^{3}$ were used. Samples were supplied by Bayer AG Leverkusen, Germany. Pieces of $2 \times 2 \mathrm{~cm}^{2}$ were irradiated with different $\gamma$-ray doses, viz. $1,10,50,75$, and $100 \mathrm{kGy}$ using $1.25 \mathrm{MeV}^{60} \mathrm{Co}$ source of dose rate $4 \mathrm{kGy} / \mathrm{h}$ at the Egyptian Atomic Energy Authority (EAEA). Pristine and $\gamma$-irradiated samples were subjected to optical measurements using UV-visible spectrometer (UVS-2700) in the wavelength range from 190 to $1100 \mathrm{~nm}$.

\section{Results and Discussion}

Figure 1 shows the UV-visible spectra in the range of 250 - $600 \mathrm{~nm}$ for pristine and $\gamma$-irradiated Makrofol DE 1-1. Pristine and $1 \mathrm{kGy}$ irradiated sample show the same behavior, while at $\gamma$-doses higher than $1 \mathrm{kGy}$ formation of color centers appeared as a shoulder superimposed on the UV spectra in the range $310-320 \mathrm{~nm}$ that may be the reason of the color change of plastic from transparent to faint yellowish 
after $\gamma$-irradiation. Figure 2 depicts the absorbance at $315 \mathrm{~nm}$ versus irradiated $\gamma$-doses for Makrofol DE 1-1 detector, it shows that the absorbance curve has a sharp increase in the range ( 0 to $10 \mathrm{kGy}$ ) and then decreases slowly (almost linearly) with regression coefficient (0.99) up to $75 \mathrm{kGy}$. Such linearity reflects the validity of the absorbance parameter to serve as a dosimeter within this dose window recorded at wavelength $315 \mathrm{~nm}$. Beyond $75 \mathrm{kGy} \gamma$-dose, absorbance shows a sharp decrease. The decrease in absorbance (see figure 2) might be due to the increase of broken $\mathrm{C}=\mathrm{C}$ bonds around the color centers at $315 \mathrm{~nm}$.

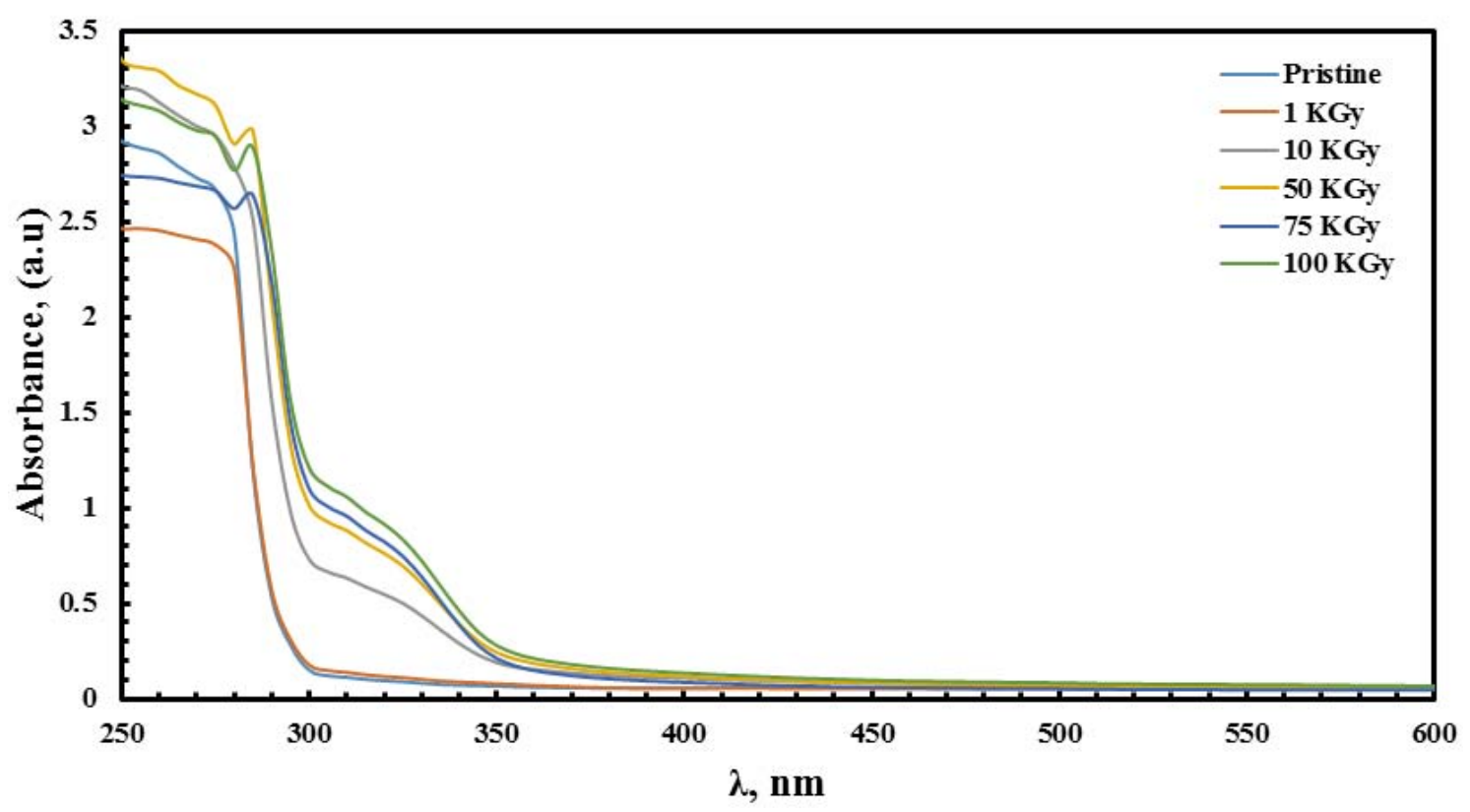

Figure 1. UV-visible Spectra of the Pristine and $\gamma$-irradiated Makrofol DE 1-1 at Different Doses.

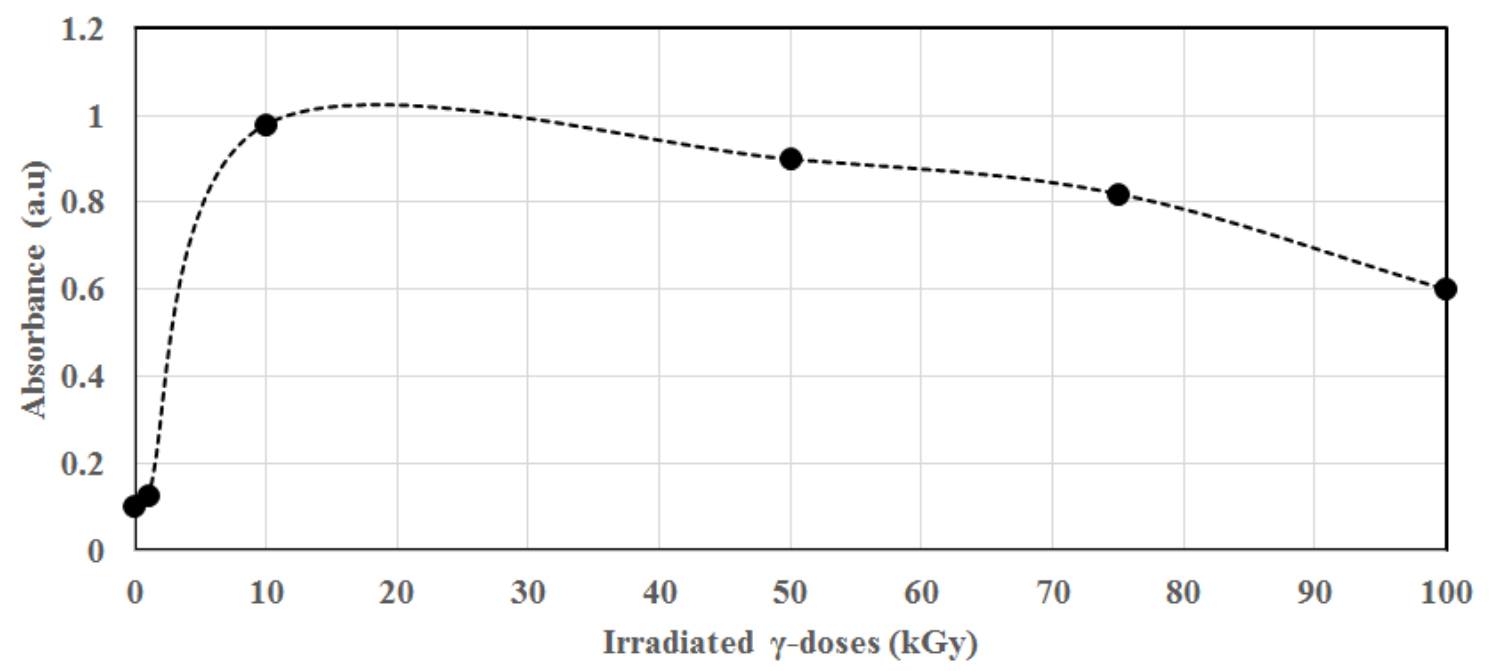

Figure 2. The Absorbance of Pristine and $\gamma$-irradiated Makrofol DE 1-1 Detectors Versus Irradiated $\gamma$-Doses at Wavelength 315 nm.

In order to calculate the optical energy band gaps for the studied samples, the coefficient of optical absorption, $\alpha$ was computed from the absorbance, $A$ data using Eq.(1) [11]:

$$
\alpha(E)=2.303 \frac{A(E)}{d}
$$

where $E=h v$ is the incident photon energy. $A(E)$ is the absorbance, which is given by $A=\log \left(I_{o} / I\right)$, where $I_{o}$ is the intensity of incident beam and $I$ is the intensity of transmitted beam. $d$ is the thickness of the sample. For non-crystalline materials the absorption coefficient can be expressed as a function of optical gap between the valance and the conduction bands $E_{\text {opt. }}^{\text {Tauc }}$ and the photons energy $E=h v$ by Tauc's [12] and modified by Davis and Mott [13] as the following relation:

$$
\alpha(v) E=G\left(E-E_{\text {opt. }}^{\text {Tauc }}\right)^{m}
$$

$\mathrm{m}$ is $1 / 2$ for direct and 2 for indirect allowed transitions [14]. $\mathrm{G}$ is a constant mainly depends on the probability of 
transition. In Tauc's model [12], the optical energy gap, $E_{\text {opt. }}^{\text {Tauc }}$ determined by plotting $(\alpha E)^{1 / m}$ versus the photon energy $(E=h v)$. The direct band gaps are determined by plotting $(\alpha E)^{2}$ versus the photon energy $(E)$, while the indirect band gaps are determined by plotting $(\alpha E)^{1 / 2}$ versus the photon energy $(E)$. The extrapolation of the straight parts of the curves to the energy axis $(E)$ yields the direct and indirect band gaps for pristine and irradiated Makrofol DE 1-
1 samples. Figures 3 and 4 depict the variation of $(\alpha E)^{1 / 2}$ and $(\alpha E)^{2}$ versus the photon energy $(E)$, respectively. The values of direct and indirect band gaps have been estimated for pristine and $\gamma$-irradiated Makrofol DE 1-1 polymer from the extrapolating of the straight regions of the obtained curves to the $E=h v$ axis. The obtained energy gap values of different transition are tabulated in Table 1.

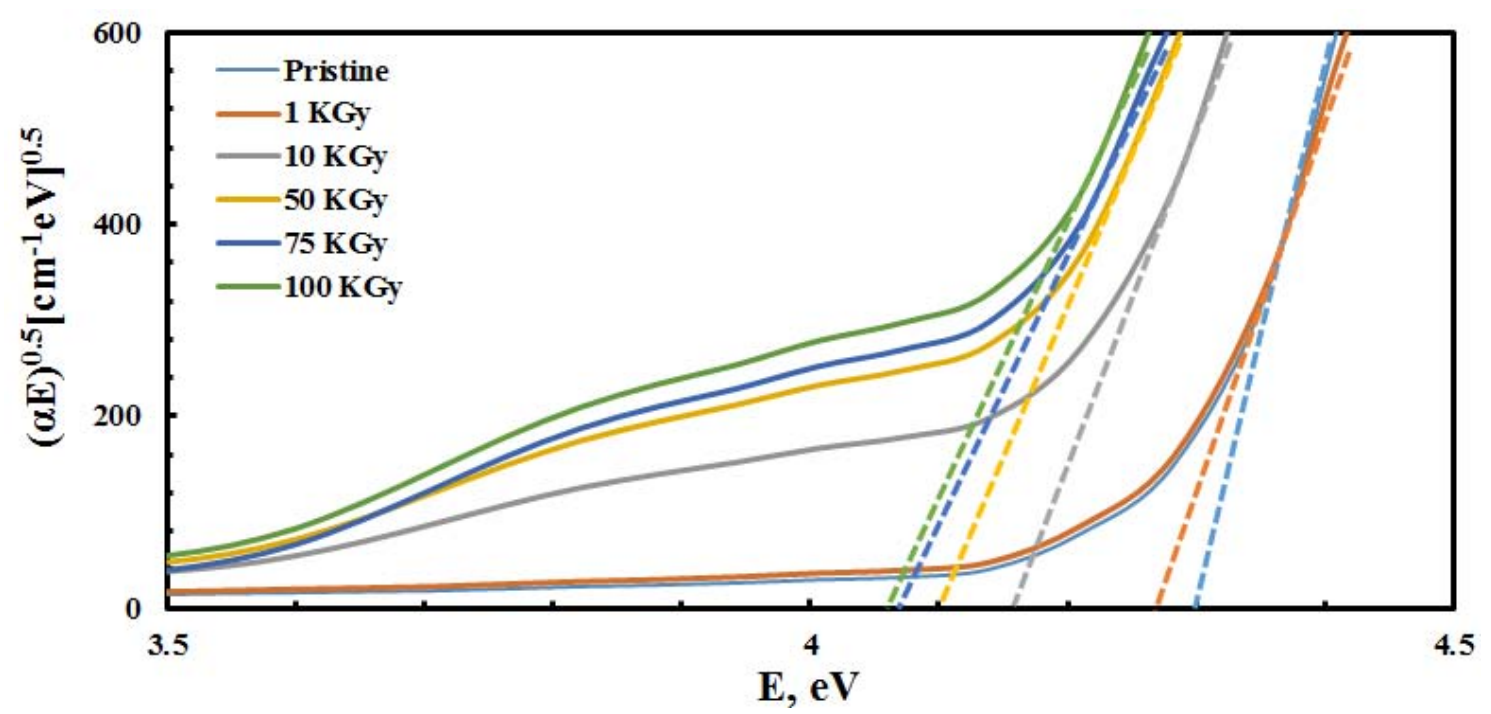

Figure 3. Plots of $(\alpha E)^{0.5}$ with (E) for Pristine and Makrofol DE 1-1 Samples Irradiated with Different Doses of Gamma Rays.

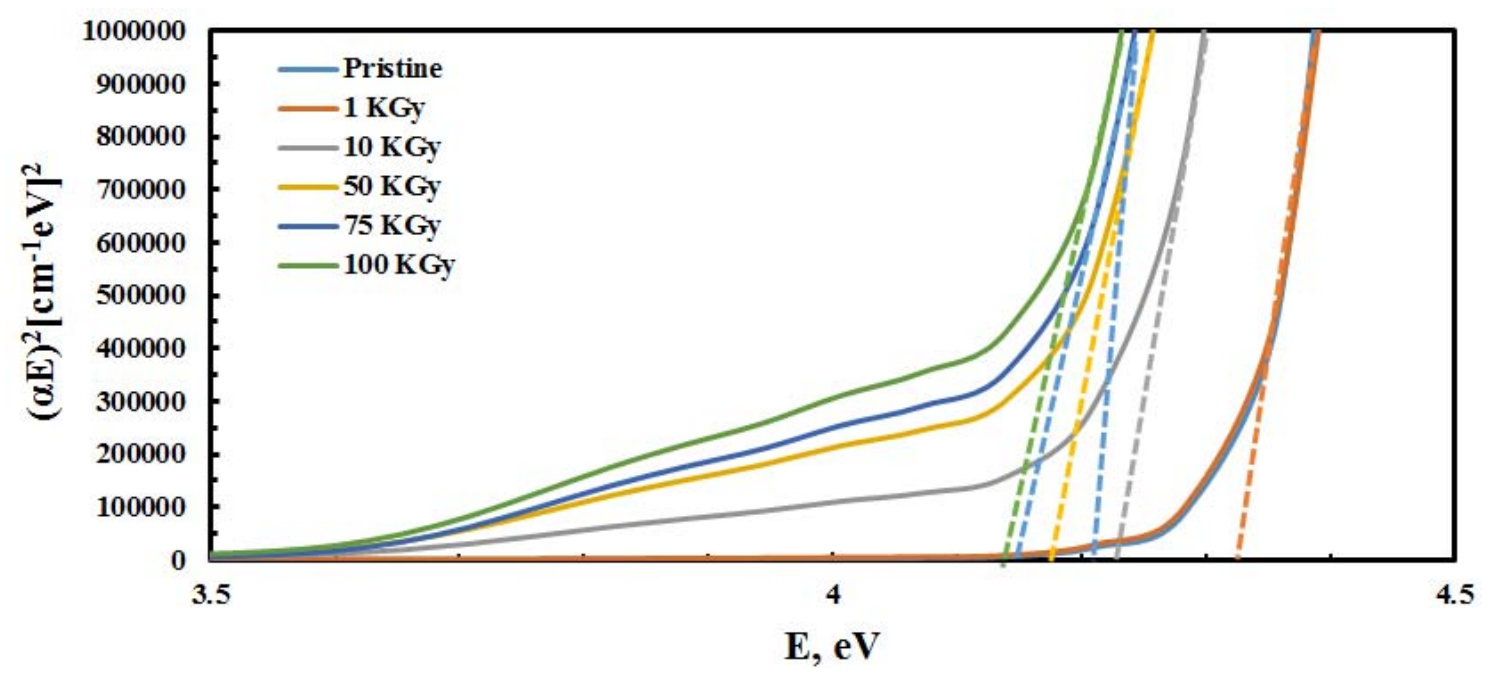

Figure 4. Plots of $(\alpha E)^{2}$ with (E) for Pristine and Makrofol DE 1-1 Samples Irradiated with Different Doses of Gamma Rays.

Table 1. E Eaut, T, M, and n for Pristine and y-irradiated Makrofol DE 1-1 Samples Obtained from Tauc's Model.

\begin{tabular}{|c|c|c|c|c|c|c|c|c|}
\hline \multirow[t]{2}{*}{$\begin{array}{l}\gamma \text {-irradiation } \\
\text { (kGy) }\end{array}$} & \multicolumn{2}{|c|}{$\begin{array}{l}\text { Optical band gap, } \mathrm{E}_{\text {opt. }}^{\text {Tauc }} \\
\text { (eV) }\end{array}$} & \multicolumn{2}{|c|}{$\begin{array}{l}\text { No. of carbon atom per } \\
\text { conjugated length }(\mathrm{N})\end{array}$} & \multicolumn{2}{|c|}{$\begin{array}{l}\text { No. of carbon atom per } \\
\text { clusters }(M)\end{array}$} & \multicolumn{2}{|c|}{ Refractive index (n) } \\
\hline & Indirect & Direct & Indirect & Direct & Indirect & Direct & Indirect & Direct \\
\hline 0 & 4.30 & 4.34 & 4.23 & 4.19 & $\sim 64$ & $\sim 62$ & 2.11 & 2.12 \\
\hline 1 & 4.22 & 4.32 & 4.34 & 4.21 & $\sim 66$ & $\sim 63$ & 2.12 & 2.11 \\
\hline 10 & 4.16 & 4.23 & 4.37 & 4.30 & $\sim 68$ & $\sim 65$ & 2.13 & 2.12 \\
\hline 50 & 4.11 & 4.18 & 4.43 & 4.35 & $\sim 70$ & $\sim 67$ & 2.14 & 2.13 \\
\hline 75 & 4.07 & 4.15 & 4.46 & 4.38 & $\sim 71$ & $\sim 68$ & 2.15 & 2.14 \\
\hline 100 & 4.06 & 4.13 & 4.48 & 5.40 & $\sim 72$ & $\sim 69$ & 2.16 & 2.15 \\
\hline
\end{tabular}


Absorption spectrum fitting (ASF) model is another method used to determine the energy band gap for polymer. This method depends on the measurement of the material absorbance only, and all calculation is avoiding the film thickness measurement which commonly could not be measured precisely. In this procedure, Eq.(2) can expressed as function of the wavelength $(\lambda)[15,16]$ :

$$
\alpha(\lambda)=G(h c)^{n-1} \lambda\left(\lambda^{-1}-\lambda_{\text {opt }}^{-1}\right)^{m}
$$

where $\lambda_{\text {opt. }}, h$ and $c$ are the wavelength corresponding to the optical gap, Planck's constant and the velocity of the light, respectively. Eq. (3) can be rewrite using the Beer-Lambert's law $[\alpha(\lambda)=(2.303 / d) A]$ as:

$$
A(\lambda)=D \lambda\left(\lambda^{-1}-\lambda_{\text {opt. }}^{-1}\right)^{m}
$$

where $D=\left[G(h c)^{m-1} d / 2.303\right]$. By Eq. (4), the optical band gap can be calculated by the absorbance spectrum fitting method without any need to the film thickness. Thus the value of band gap, in $\mathrm{eV}$, can be determined from the parameter $\lambda_{\text {opt. }}$ using,

$$
E_{\text {opt. }}^{A S F}=\frac{h c}{\lambda_{\text {opt. }}}=\frac{1239.83}{\lambda_{\text {opt. }}}
$$

The value of $\lambda_{\text {opt. }}$ can be determined by extrapolating the linear part of $(\mathrm{A} / \lambda)^{1 / \mathrm{m}}$ versus $(1 / \lambda)$ curve at $(\mathrm{A} / \lambda)^{1 / \mathrm{m}}=0$. Using the optical absorption spectra of pristine and $\gamma$ irradiated Makrofol DE 1-1 detector samples (shown in figure 1$)$, the plots of $(\mathrm{A} / \lambda)^{1 / 2}$ and $(\mathrm{A} / \lambda)^{2}$ versus $(1 / \lambda)$ were drawn as shown in figures 5 and 6 , respectively. The values of $E_{\text {opt. }}^{A S F}$ are collected in Table 2.

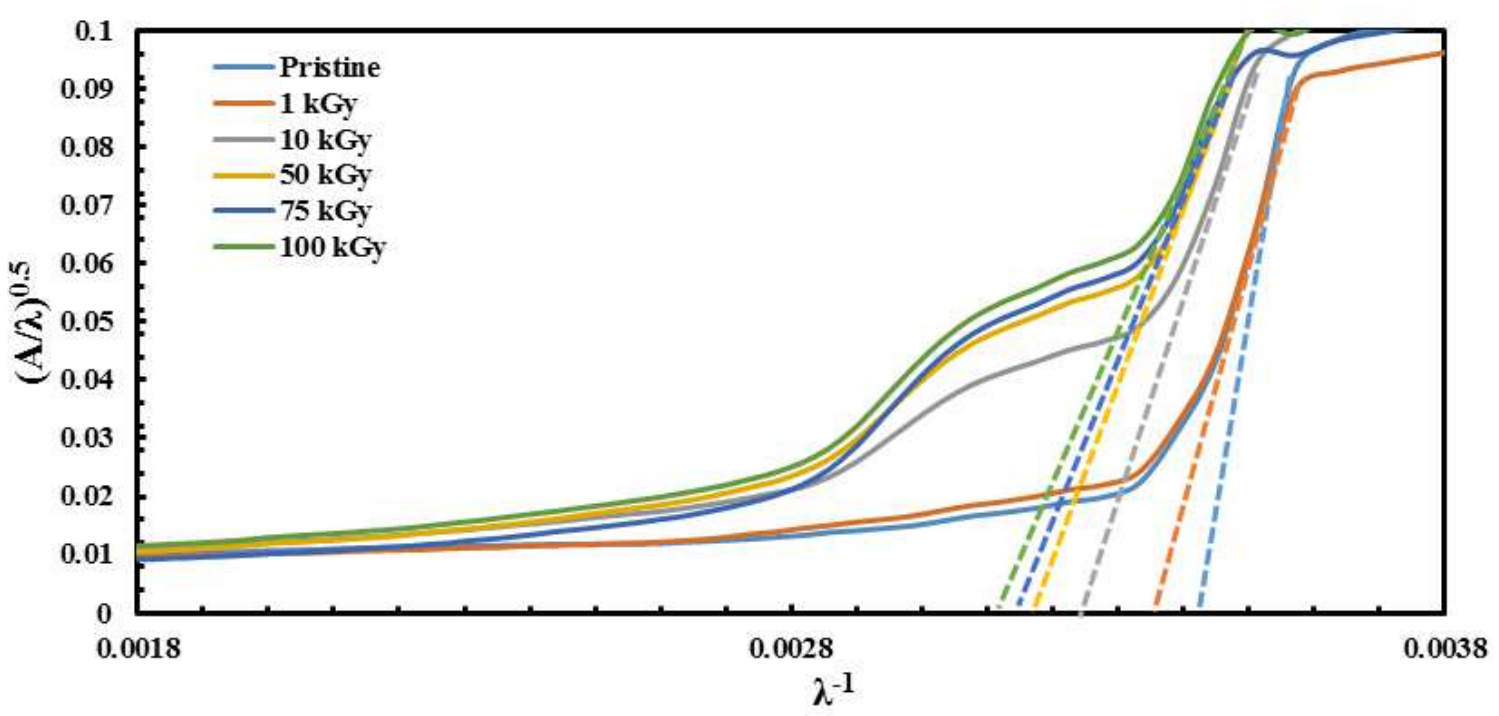

Figure 5. Plots of $(A / \lambda)^{0.5}$ with $\left(\lambda^{-1}\right)$ for Pristine and Makrofol DE 1-1 Samples Irradiated with Different Doses of Gamma Rays.

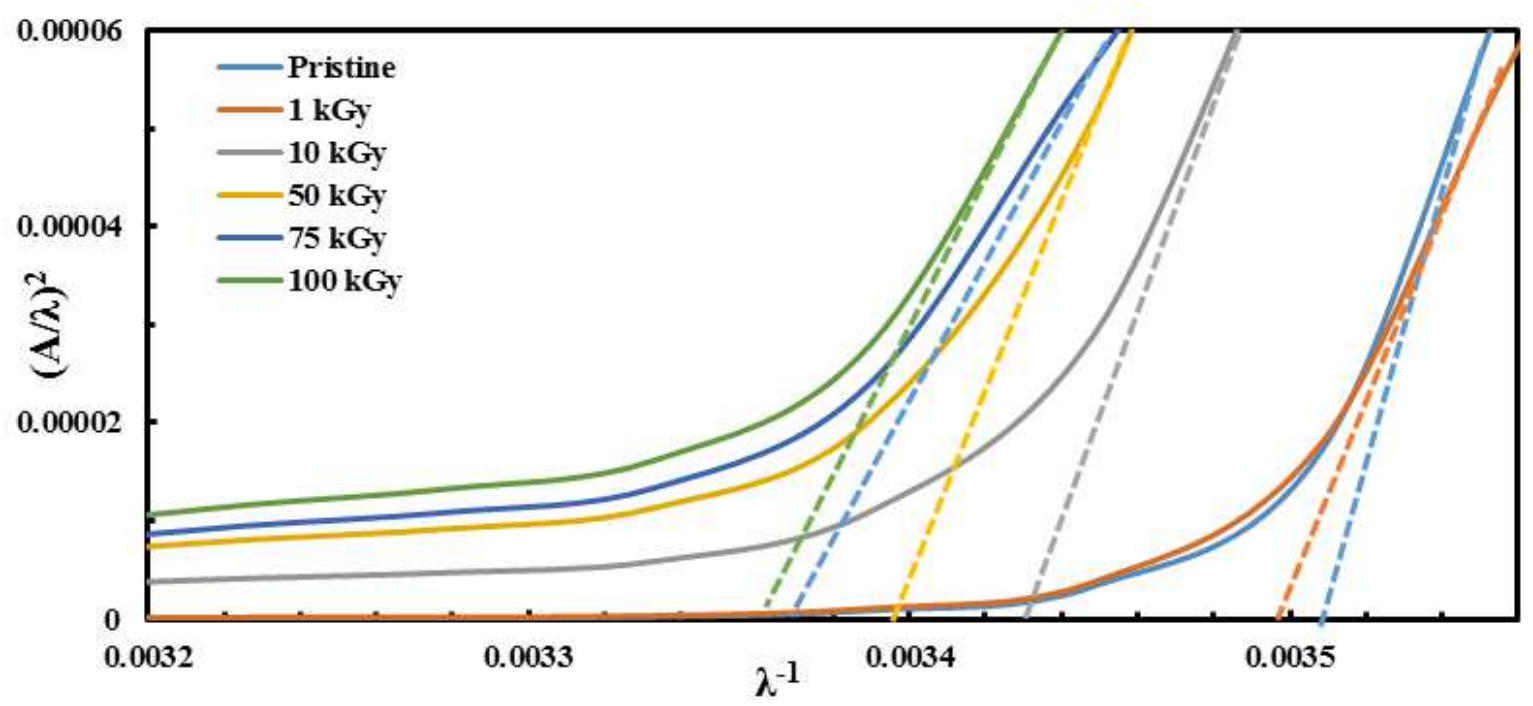

Figure 6. Plots of $(A / \lambda)^{2}$ with $\left(\lambda^{-1}\right)$ for Pristine and Makrofol DE 1-1 Samples Irradiated with Different Doses of Gamma Rays. 
Table 2. $E_{\text {opt. }}^{A S F}, N, M, n$ for Pristine and $\gamma$-irradiated Makrofol DE 1-1 Samples Obtained from ASF Model.

\begin{tabular}{|c|c|c|c|c|c|c|c|c|}
\hline \multirow[t]{2}{*}{$\begin{array}{l}\gamma \text { - irradiation } \\
(\mathrm{kGy})\end{array}$} & \multicolumn{2}{|c|}{ Optical band gap $\mathrm{E}_{\text {opt. }}^{\mathrm{ASF}}(\mathrm{eV})$} & \multicolumn{2}{|c|}{$\begin{array}{l}\text { No. of carbon atom per } \\
\text { conjugated length }(\mathbf{N})\end{array}$} & \multicolumn{2}{|c|}{ No. of carbon atom per clusters (M) } & \multicolumn{2}{|c|}{$\begin{array}{l}\text { Refractive index } \\
\text { (n) }\end{array}$} \\
\hline & Indirect & Direct & Indirect & Direct & Indirect & Direct & Indirect & Direct \\
\hline 0 & 4.24 & 4.33 & 4.29 & 4.19 & $\sim 65$ & $\sim 62$ & 2.12 & 2.10 \\
\hline 1 & 4.14 & 4.32 & 4.39 & 4.21 & $\sim 69$ & $\sim 63$ & 2.14 & 2.11 \\
\hline 10 & 3.99 & 4.24 & 4.56 & 4.29 & $\sim 74$ & $\sim 65$ & 2.17 & 2.12 \\
\hline 50 & 3.90 & 4.19 & 4.66 & 4.34 & $\sim 77$ & $\sim 67$ & 2.18 & 2.13 \\
\hline 75 & 3.84 & 4.15 & 4.74 & 4.38 & $\sim 80$ & $\sim 68$ & 2.20 & 2.14 \\
\hline 100 & 3.80 & 4.14 & 7.78 & 4.39 & $\sim 81$ & $\sim 69$ & 2.21 & 2.14 \\
\hline
\end{tabular}

The values of energy gaps which obtained from Tacu's and ASF models indicate the simultaneous existence of indirect and direct band gap in Makrofol DE 1-1 detector with decreasing tendency at higher $\gamma$ - irradiation dose. Furthermore, the values of indirect energy gap have been found to be lower than the corresponding values for the direct energy gap as shown in Tables 1 and 2 which illustrated in figure 7 . The decrease in the optical energy values with increasing $\gamma$-irradiation dose is due to formation of carbon enriched clusters due to partial evolution of hydrogen molecules [17]. These decreases may be attributed to irradiation induced defects and/or increase the number of conjugated bonds $(-\mathrm{C}=\mathrm{C}-$ ). In other word, the conductivity of the bombarded polymer surface with $\gamma$-irradiation is greater than that of the bulk sample. This confirms that the ion beam technique can be used in fabricating electronic devices. In addition, such a decrease in $E_{\text {opt. }}^{\text {Tauc }}$ may be attributed to the formation of new photo-chemicals, which form the trap levels between the HOMO and LUMO energy states, making the lower energy transitions feasible and results in the reduction of the optical gap.

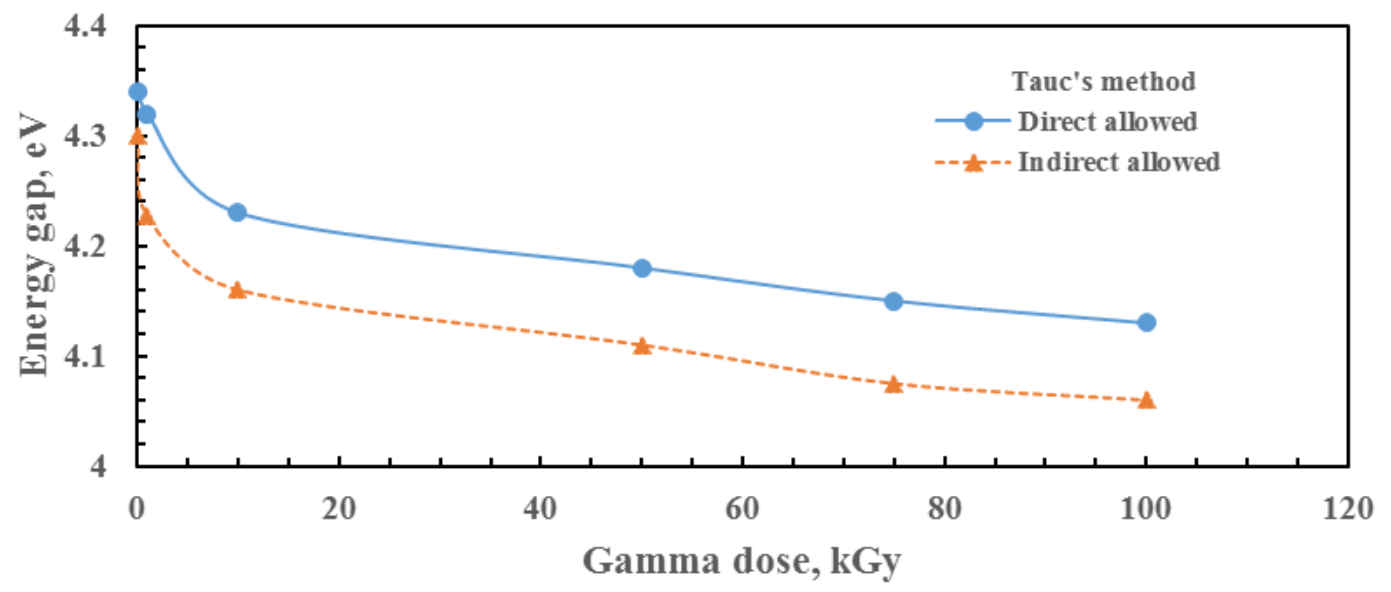

(a)

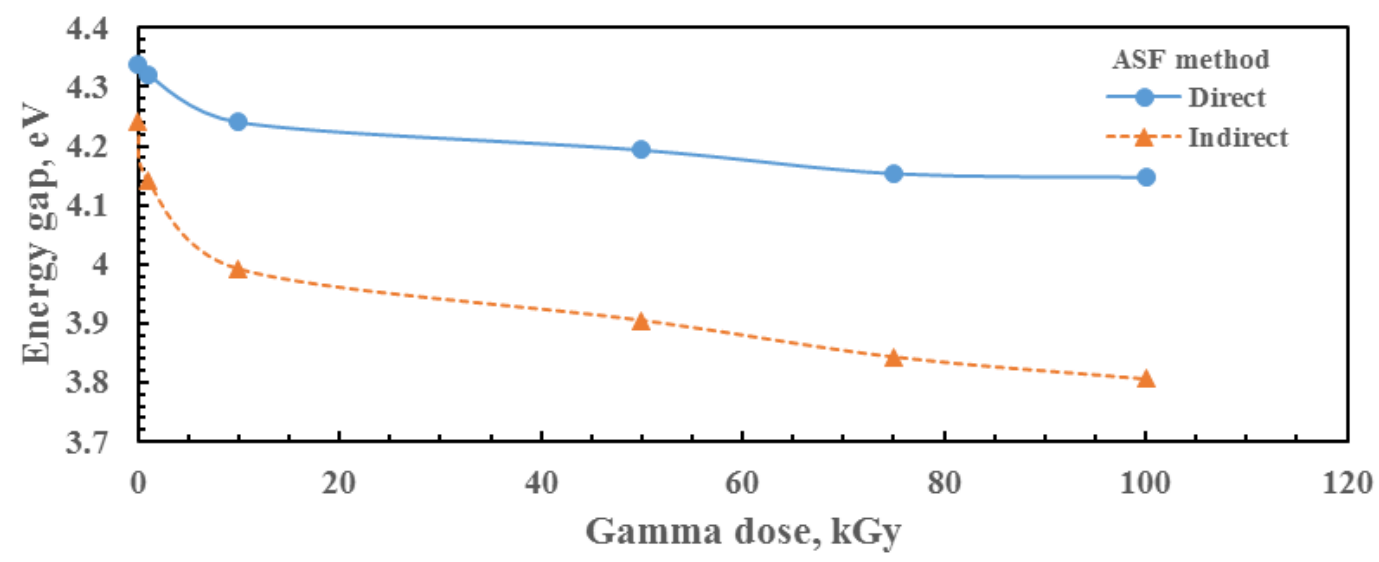

(b)

Figure 7. Dependence of the Optical Energy Gap $E_{\text {opt. }}(\mathrm{eV})$ on Gamma Dose (kGy) for Pristine and Makrofol- DE 1-1 Samples Irradiated with Different Gamma Doses (a) in Tauc's method. (b) in ASF Method. 
The obtained values of the energy gaps for indirect and direct transition in both Tauc's and ASF models were used in order to calculate the refractive index, $\mathrm{n}$ of the present samples using Eq.(6) [18]:

$$
\left(\frac{n^{2}-1}{n^{2}+2}\right)=1-\sqrt{\frac{E_{\text {opt }}}{20}}
$$

The values of refractive index, $\mathrm{n}$ for the pristine and $\gamma$ irradiated samples are collected in Tables 1 and 2. The refractive index values direct band gap less than that for the indirect energy band gap, and increases with increasing of $\gamma$ dose.

Makrofol DE 1-1 detector is an aromatic polymer characterized by a linear chain structure. The shift in the absorption edges towards the visible from the ultraviolet (see figure1) could be related to an increase in the conjugation length and a condensation of the aromatic rings into a compact carbonaceous cluster. Thus, the No. of carbon atoms per conjugation length, $\mathrm{N}$ and the No. of carbon atoms per cluster, $M$ are important parameters that enhance the optical properties of the polymeric material. This is due to these parameters rich with charge carrier, which enhances the surface conductivity of the polymers. For a linear structure, $\mathrm{N}$, is given by [19]:

$$
N=\frac{2 \beta \pi}{E_{\text {opt }}}
$$

$\beta=-2.9 \mathrm{eV}$ as it is associated with $\pi-\pi^{*}$ optical transitions in $-\mathrm{C}=\mathrm{C}-$ structure. $2 \beta$ gives the band structure energy of a pair of adjacent $\pi$ sites. $M$, is given by [19]:

$$
M=\left(\frac{34.3}{E_{o p t}}\right)^{2}
$$

$N$ and $M$ for indirect and direct transition were calculated using energy gap values which obtained in both Tauc's and ASF models and listed in Tables 1 and 2. It is clear that $N$ and $M$ for the direct band gap lower than that for the indirect energy band gap, and their values increase with increasing of the irradiated $\gamma$-dose. This probably attributed to cleavages of $\mathrm{C}-\mathrm{H}$ bonds during the irradiation and, as a consequence, to the release of hydrogen.

\section{Conclusion}

UV-vis spectra of pristine and $\gamma$-irradiated Makrofol DE 11 polymer detector reveal a shift toward the longer wavelength with increasing of $\gamma$-irradiated dose. Tauc's and the absorption spectrum fitting (ASF) models were used to calculate the optical energy gaps for the studied samples.
Direct and indirect energy gaps of are correlated with $\gamma$-dose, decreasing with increasing of irradiation dose. This indicates that the $\gamma$-irradiated samples leads to the appearance of the energy states that are induced upon irradiation and depend on the irradiation dose. The results $\mathrm{E}_{\text {opt. }}$ show a decrease with increasing of $\gamma$-irradiated doses as estimated from the linear part of optical spectra. Meanwhile $(\mathrm{N})$ and $(\mathrm{M})$ raise when $\gamma$ irradiated dose increase.

\section{References}

[1] Hussein, A., Shinishen, Kh., Abou El-Kheir, A. A., 1993. J. Mater. Sci. 28, 5138.

[2] Hussein, A., Hager, S. A., El-Nimr, M. K., Ghanim, E., H., 1994. Nucl. Instr. Meth. Phys. Res. B 88, 490.

[3] El-Samman, H., Mansy, M., Hussein, A., El-Hawary, M., ElSersy, A.,1999. Nucl. Instr. Meth. B 155,426.

[4] Kumar, A., Singh, B., Singh, S., 2001. Ind. J. Pure Appl. Phys. 39,761 .

[5] El-Sersy, A., El-Samman, H., Hussein, A., El-Hawary, M., 2004. Radiat. Phys. Prot. Con. Nov., Egypt.

[6] Yu, K., N., Leung, S., Y., Y., Nikezic, D., Leung, J., K., C., 2008. Radiat. Meas. 43, 357.

[7] Yu, K., N., Nikezic, D., 2012. Appl. Radiat. Iso. 70, 1104.

[8] Kumar, A., Jain, R., K., Yadav, P., Chakraborty, R., N., Singh, B. K., Nayak, B., K., 2013. J. Radio. Nucl. Chem. 295, 95.

[9] Koesis, Z., S., Dwivedi, K., K., Brandt, R., 1997. Radiat. Meas. 28, (1-6), 177.

[10] Seguchi, T., Yagi, T., Ishikawa, S., Sano, Y., 2002. Radiat. phys. chem. 63,35 .

[11] Fox, M., 2001. Optical Properties of Solids, Oxford University Press Inc., New York.

[12] Tauc, J., 1974. Amorphous and Liquid Semiconductors, in: J. Tauc (Ed.), Plenum Press, New York.

[13] Mott, N. F., Davies, E. A., 1979. Electronic Processes in NonCrystalline Materials, Clarendon Press, Oxford.

[14] Singh, S., Prasher, S., 2004. The etching and structural studies of gamma irradiated induced effects in CR-39 plastic track recorder. Nucl. Instrum. Meth. B, 222, 518-24.

[15] Alarcon, L. E., Arrieta, A., Camps, E., Muhl, S., Rudil, S., Santiago, E. V., 2007. Appl. Surf. Sci. 254, 412-415.

[16] Souri, D., Tahan, Z. E., 2015. Appl. Phys. B 119, 273-279.

[17] Rizk, M., Abdul-Kader, A. M., Alib, Z. I., Ali, M., 2009. Vacuum 83, 805.

[18] Dimitrov, V., Sakka, S., 1996. J. Appl. Phys. 79, 1736-1740.

[19] Nikezic, D., Yu, K. N., 2004. Mater. Sci. Eng. 46, 51. 